\section{Classical Wegener’s as Non Resolving Pneumonia}

Pragati Rao D*, Kalyani K, Venkateswara Rao P, and Sreelatha V

Department of Pulmonary Medicine, NRI General Hospital, Chinakakani, Guntur, Andhra Pradesh, India

\begin{abstract}
A $32 \mathrm{yr}$ old female clinically and radiographically diagnosed as tuberculosis initially not responding to anti tuberculous treatment was later on Diagnosed as classical Wegener's granulomatosis basing on tissue biopsy, c-ANCA, along with other systemic manifestations posing a diagnostic challenge.
\end{abstract}

Keywords: Wegener's granulomatosis (WG); Non resolving pneumonia; Renal biopsy; C-ANCA

\section{Key Messages}

High index of suspicion in non resolving pneumonia. Early diagnosis and prompt treatment of Wegener's granulmatosis as disease progresses rapidly with renal involvement. Drugs used to treat WG are highly toxic, so definitive diagnosis is essential.

\section{Introduction}

Wegeners Granulomatosis (WG) is an uncommon multisystemic disease of auto immnune origin characterised by necrotising granulomatous vasculitis of small calibre vessels [1].Clinical presentation is variable. Classical triad involving upper and lower respiratory tract and kidneys with other organs like ocular, cutaneous,rheumatological, neural, gastrointestinal and lower genitourinary systems.

Pulmonary and upper respiratory tract involvement occurs early. Involvement of lungs has been reported in up to $94 \%$ of patients [2]. It may present with isolated pulmonary symptoms [3] with chest radiographs showing nodular leison of varying sizes, which may later cavitate (50\%) consolidation of air space, ground glass opacification secondary to pulmonary hemorrhage, pleural effusion (10\%), hilar and mediastinal lymph nodes (2-15\%), airway narrowing leading to collapse.

We describe this case of WG as its was first thought to be tuberculosis and its diagnosis could not be made untill other systemic manifestation and renal failure were present posing a diagnostic challenge.

\section{Case History}

A 32 yr old female presented with complaints of high grade fever, cough with scanty expectoration, She was on ATT since 5 months, had underwent cardiac surgery for large ASD 2 months back. On examination she had a temperature of $101 \mathrm{~F}$, pulse rate of $120 / \mathrm{min}$, BP: 120/80 mm Hg, RR: 22/min, SPO2 on Room air: 98\%. On general examination pallor was present. Chest examination showed surgical scar over parasternal area with decreased air entry and occasional crepitations in right lower lobe area.

\section{Initial investigations revealed}

Chest Xray PA view (Figure 1)-consolidation patches in right mid and lower lung zones. HB-8.9 gms\%; Total count-12,700 cells/ $\mathrm{mm}^{3}$; DC-N83 L13 E01M03; ESR-130 mm/hr; Bleeding time2'00"; Clotting time-4'00"; Peripheral blood smear- Hypochromic microcytic and predominantly normocytic anemia, neutrophilic leucocytosis. Widal test-negative; Complete urine analysis-normal; Blood for culture sensitivity-negative; Viral markers-negative; Sputum for AFB-negative; Sptum for grams stain-plenty of pus cells and budding yeast cells with pseudo hyphae seen. Sputum for $\mathrm{c} / \mathrm{s}-$ candida species isolated; Blood grouping and typing-B+ve. She was

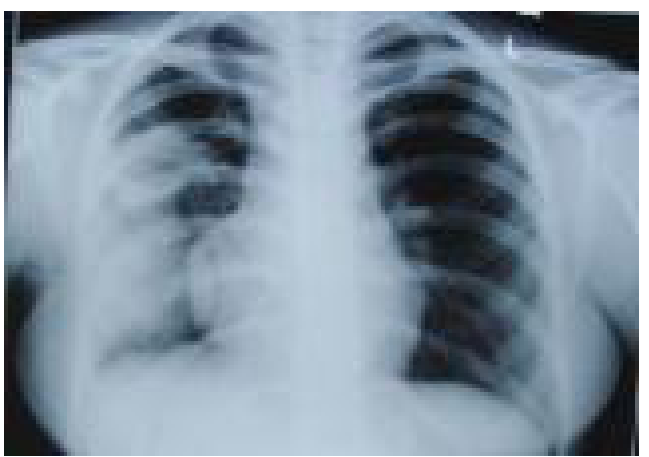

Figure 1: On admission with consolidation on right side.

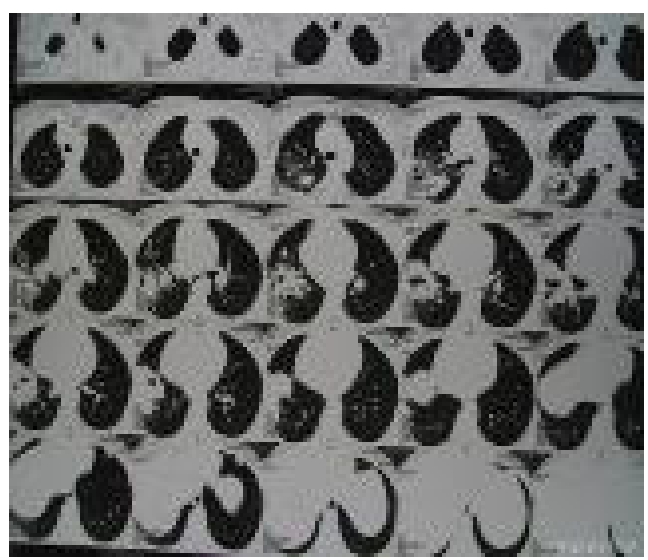

Figure 2: HRCT chest with thick walled cavities and adjacent consolidation.

started on IV antibiotics cefoperazone+sulbactum, tab fluconazole $150 \mathrm{mg}$ od. Her ATT was continued. Anaemia was corrected with oral iron. There was no clinical response and her antibiotic regimen was changed to clarithromycin oral; IV amikacin and was discharged on request.

*Corresponding author: Pragati Rao D, Department of Pulmonary Medicine NRI General Hospital, Chinakakani, Guntur, Andhra Pradesh, India, E-mail: kalyanimedic@gmail.com

Received January 27, 2012; Accepted February 28, 2013; Published March 04 2013

Citation: Pragati Rao D, Kalyani K, Venkateswara Rao P, Sreelatha V (2013) Classical Wegener's as Non Resolving Pneumonia. J Pulmon Resp Med 3: 141. doi:10.4172/2161-105X.1000141

Copyright: () 2013 Pragati Rao D, et al. This is an open-access article distributed under the terms of the Creative Commons Attribution License, which permits unrestricted use, distribution, and reproduction in any medium, provided the original author and source are credited. 
On revisit after 10 days, fever persisted with chest X-ray revealing no change in the opacities. She was advised HRCT which suggested (Figure 2): Thick walled irregular cavitary lesion seen at right mid zone with an adjacent pneumonic patch, Right loculated pleural effusion.

She developed conjunctivitis, arthralgia, and rash over nose and nasal septum, glossitis, which she declared as recurrence. As the pneumonia was not resolving she was further evaluated (Table 1).

In view of deranged renal function, renal biopsy was done and C-ANCA levels were sent, suspecting pulmonary renal syndrome. Renal biopsy revealed crescentic glomerulo nephritis (9/13) with significant immune deposits on IF studies consistent with pauci immune glomerulo nephritis.C-ANCA (pr3)-136.64 $\mu / \mathrm{ml}$ (6 times normal).

A diagnosis of Wegeners's was made. She was started on IV methyl prednisolone and cyclophosphamide. Dramatic response was seen. Her urea creatinine levels reached normal levels with resolution of pulmonary infiltrates.

\section{Discussion}

The diagnosis of WG in this patient was challenging due to its atypical Presentation as non resolving pneumonia [4]. India is an endemic country for tuberculosis, first diagnosis to be considered is pulmonary tuberculosis.

A non resolving pneumonia with systemic manifestations, pulmonary renal syndrome [5] and renal biopsy along with c-ANCA [6], is a clue to final diagnosis as WG.

Diagnosis of WG is based on typical clinical and imaging features associated with pr 3-anca. A set of four clinical criteria including an abnormal urine sediment, abnormal chest X-ray, Oral ulcers and nasal discharge, granulomatous inflammation on biopsy were used in current clinical practice for diagnosis of WG. These criteria are $88 \%$ sensitive and $92 \%$ specific.

WG is a systemic small vessel vasculitis first described by winger in 1933, followed by Wegener in 1936, and Ringerts in 1947. It is a relatively uncommon disease with mean age of presentation 50yrs with equal male to female ratio. Onset is acute or sub acute but may be

\begin{tabular}{|c|c|}
\hline Test & Result \\
\hline Bronchoscopy and BAL & $\begin{array}{l}\text { No endo bronchial lesion present. BAL was negative } \\
\text { for AFB, fungi, Malignant cells }\end{array}$ \\
\hline \multirow{4}{*}{ Urine analysis } & Albumin-++ \\
\hline & Pus cells-10-15/HPF \\
\hline & RBC-plenty \\
\hline & Urine Culture-sterile \\
\hline \multirow{5}{*}{ Renal function test } & Blood urea-77 mg/dl \\
\hline & Serum creatinine $-4.7 \mathrm{mg} / \mathrm{dl}$ \\
\hline & Serum uric acid-5.8 mg/dl \\
\hline & Sodium-123 mmol/l \\
\hline & Potassium-3 mmol/l \\
\hline C reactive protein & Negative \\
\hline RA factor & Negative \\
\hline ANA levels & Negative \\
\hline \multirow{2}{*}{ Ultra sound abdomen } & Renal parenchymal changes? ATN \\
\hline & $\begin{array}{l}\text { Orthopaedic Examination-B/L knee joint effusion+, } \\
\text { B/L synovial thickening }\end{array}$ \\
\hline $\begin{array}{l}\text { OTO Laryngeal } \\
\text { examination }\end{array}$ & $\begin{array}{l}\text { A single pale granulomatous leison over post } 1 / 3 \mathrm{rd} \\
\text { of vocal cords }\end{array}$ \\
\hline
\end{tabular}

Table 1: Investigations \& Results on further evaluation after readmission.

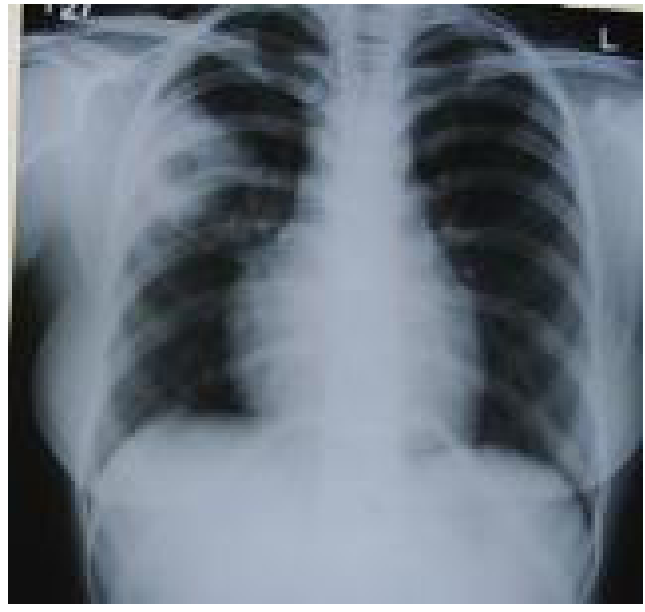

Figure 3: Early in the disease.

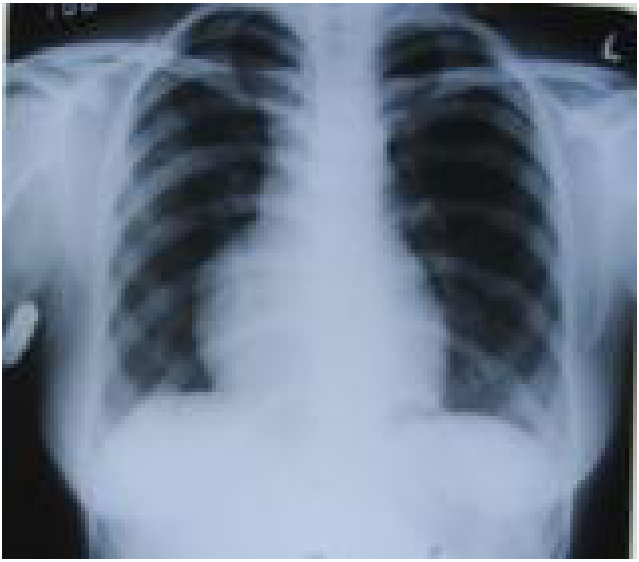

Figure 4: After initiation of treatment.

indolent. At presentation upper airway involvement is most common and manifest as sinusitis and nasal ulceration, otitis pain and hearing loss. Larynx and sub glotticregion is involved causing hoarseness and dyspnoea. Pulmonary involvement typically involves all the three components of lung parenchyma, air ways, and pleura. Radiographic changes occur in lungs in 50-75\% at presentation.

Functional renal impairment is unusual at presentation being seen only in only $10 \%$ with Glomerulonephritis associated hematuria and proteinuria and can lead to renal failure if not treated aggressively. About $85 \%$ with multi organ involvement have pr3-anca positivity with sensitivity of $91 \%$ and specificity of $99 \%$ for active disease. The interesting point to be noted is individual pulmonary opacities with or without cavitation decrease in size or even disappear before therapy [7] which was apparent in this case on following serial X-rays (Figures 3 and 4).

Other systems like musculoskeletal system may involve at onset in $30 \%$ cases with arthralgias, arthritis, myalgias acute myositis and jaw claudication. Central nervous system is involved in $20 \%$ cases may manifest as Peripheral neuropathy, Central neuropathy, External opthalmoplegia, CVA, Seizures, Cerebritis. Peripheral nervous system involvement typically occurs as mononeuritis multiplex or polyneuritis. Eye manifestations occur in 10-15\% as Conjunctivitis, Dacrocystitis, Scleritis, Proptosis, Visual loss, Diplopia. Heart 
involvement is uncommon and may cause congestive heart failure, cardiomyopathy and pericariditis, which is a result of vascular occlusion. Skin lesions like palpable purpura, pyoderma like ulcers, papules, petechiae, nodules, bullae and gangrene are seen at the onset in $10-15 \%$ cases.

A limited form of disease has also been described affecting lungs with or without upper respiratory disease but without renal and other systemic involvement' early diagnosis and prompt treatment is crucial, which is often delayed. Disease progresses rapidly without treatment. Untreated WG carries a poor prognosis with death occurring in about 5 months from renal failure. The drugs used to treat WG are highly toxic, a definitive diagnosis is therefore very essential. Once renal involvement is noticed the treatment should be very aggressive.

Treatment of WG is Prednisone initiated at $1 \mathrm{mg} / \mathrm{kg}$ daily for 1 to 2 months then tapered. Cyclophosphamide $2 \mathrm{mg} / \mathrm{kg}$ daily for at least 12 months, $>90 \%$ improve and $75 \%$ remit. For the maintenance of remission switch over to azathioprine $2 \mathrm{mg} / \mathrm{kg} /$ day and to reduce toxicity of Cyclophoshamide there is some evidence that long term cotrimoxazole which reduces relapse rate.

\section{References}

1. Frankel SK, Cosgrove GP, Fischer A, Meehan RT, Brown KK (2006) Update in the diagnosis and management of pulmonary vasculitis. Chest 129: 452-465.

2. Jolly M, Molta C, Hoffman G (2000) Wegener's granulomatosis: pitfalls in the management of pulmonary disease: A case of Wegener's granulomatosis with a hilar mass. J Rheumatol 27: 2511-2512.

3. Fishman AP, Fishman JA (2008) Fishmans pulmonary diseases and disorders (4thedn), Mc Graw Hill Companies, USA.

4. http://www.eric.vcu.edu/pub/MR/MR_20051202_ramachandran.pdf

5. http://cdn.intechopen.com/pdfs/22835/InTechDifferential_diagnosis_of_the pulmonary_renal_syndrome.pdf

6. Finkielman JD, Lee AS, Hummel AM, Viss MA, Jacob GL, et al. (2007) ANCA are detectable in nearly all patients with active severe Wegener's granulomatosis. Am J Med 120: 643.

7. Fraser RS (1999) Fraser and Pares Diagnosis of diseases of chest (4thedn), W.B.Saunders company, USA. 